\title{
Online/Offline Identity-Based Signcryption Revisited $^{\star}$
}

\author{
Joseph K. Liu, Joonsang Baek, and Jianying Zhou \\ Cryptography and Security Department \\ Institute for Infocomm Research $\left(\mathrm{I}^{2} \mathrm{R}\right)$, Singapore \\ $\{k s l i u, j$ sbaek, jyzhou\}@i2r.a-star.edu.sg
}

\begin{abstract}
In this paper, we redefine a cryptographic notion called $\mathrm{On}$ line/Offline Identity-Based Signcryption. It is an "online/offline" version of identity-based signcryption, where most of the computations are carried out offline while the online part does not require any heavy computations such as pairings or multiplications on elliptic curve. It is particularly suitable for power-constrained devices such as smart cards. We give a concrete implementation of online/offline identity-based signcryption, which is very efficient and flexible. Unlike all the previous schemes in the literature, our scheme does not require the knowledge of receiver's information (either public key or identity) in the offline stage. The receiver's identity and the message to be signcrypted are only needed in the online stage. This feature provides a great flexibility to our scheme and makes it practical to use in real-world applications. To our knowledge, our scheme is the first one in the literature to provide this kind of feature. We prove that the proposed scheme meets strong security requirements in the random oracle model, assuming the Strong Diffie-Hellman (SDH) and Bilinear Diffie-Hellman Inversion (BDHI) are computationally hard.
\end{abstract}

\section{Introduction}

\subsection{Motivation}

Providing efficient mechanisms for authentication and confidentiality is probably the most important requirement in electronic transactions, especially in mobile devices or smart cards. Since attackers can easily access the physical layer and launch some potential attacks in such devices, inclusion of cryptographic protection as a countermeasure should be very effective. However, due to the power-constrained nature of these devices, only light operations are allowed to be implemented. For this reason, efficiency becomes the main concern in the design of cryptographic algorithm for such environment.

IDENTITY-BASED CRYPTOGRAPHY. Identity (ID)-based Cryptography, introduced by Shamir [18], eliminates the need for checking the validity of certificates

* This work is funded by the A*STAR project SEDS-0721330047. 
in traditional public key infrastructure (PKI). In an ID-based cryptography, public key of each user is easily computable from an arbitrary string corresponding to this user's identity (e.g. an email address, a telephone number, and etc.). Using its master key, the private key generator (PKG) then computes a private key for each user. This property avoids the requirement of using digital certificates (which contain Certificate Authority (CA)'s signature on each user's public key) and associates implicitly a public key (i.e. user identity) to each user within the system. One only needs to know the recipient's identity in order to send an encrypted message to him. It avoids the complicated and costly certificate (chain) verification for the authentication purpose. In the case of signature schemes, verification takes only the identity together with the message and a signature pair as input and executes the algorithm directly. In contrast, the traditional PKI needs an additional certification verification process, which is in fact equivalent to the computation of two signature verifications.

We argue that ID-based cryptography is particularly suitable for smart cards. The most important reason is that it eliminates the costly certificate verification process and the storage of the lengthy certificate. In addition, when there is a new card issued, other terminals or payment gateways do not need to have its certificate verified in order to communicate in a secure and authenticated way. This can greatly reduce communication overhead and computation cost.

SigncRYPTION. Signcryption, whose concept was introduced by Zheng [23], is a cryptographic primitive aiming to provide unforgeability and confidentiality simultaneously as typical signature-then-encryption technique does but with less computational complexity and lower communication cost. Due to the efficiency one can obtain, signcryption is suitable for many applications which require secure and authenticated message delivery using resource-constrained devices.

The idea of ID-based signcryption was first proposed by Malone-Lee [16]. It was further improved in $[7,13,2,8]$ for efficiency and security.

Online/Offline Signature. Online/Offline Signature was first introduced by Even, Goldreich and Micali [9]. The main idea is to perform signature generation in two phases. The first phase is performed offline (before the message to be signed is given) and the second phase is performed online (after the message to be signed is given). Online/offline signature schemes are useful, since in many applications the signer has a very limited response time once the message is presented, but he can carry out costly computations between consecutive signing requests. We note that smart card applications may take full advantages of online/offline signature schemes: The offline phase is implemented during the card manufacturing process, while the online phase uses the stored result of the offline phase to sign actual messages. The online phase is typically very fast, and hence can be executed efficiently even on a weak processor.

Online/Offline Signcryption. The notion of online/offline signcryption was first introduced by An, Dodis and Rabin [1]. As in the case of online/offline signature schemes, online/offline signcryption schemes should satisfy a basic property 
that online computation should be performed very efficiently. All expensive operations such as exponentiation or pairing computation should be conducted offline in the first phase of the scheme. Similar to online/offline signature, it is also reasonable to assume that the offline operations are independent of the particular message to be signed and encrypted, since the message only becomes available at a later stage. The second phase is performed online, once the message is presented.

An, Dodis and Rabin [1] did not give any concrete construction of online/offline signcryption, but focused mainly on establishing formal security model for signcryption and analysis of some generic constructions. The first concrete online/offline signcryption scheme was given by Zhang, Mu and Susilo [22], and it requires an additional symmetric key encryption scheme to achieve confidentiality. Another scheme can be found in [21]. However, its practicality is dubious since the scheme requires every user to execute a key exchange protocol with the remaining users in the system. Moreover, both of them are in the PKI (non ID-based) setting. The first ID-based online/offline signcryption scheme was given by Sun, Mu and Susilo [19] in a semi-generic setting, from any ID-based signature scheme.

\subsection{Limitation of existing schemes}

All of the schemes mentioned above have a restriction which renders them impractical in many situations: They require the receiver's public key / identity to be known in the offline phase, which can result in serious performance degradation. Smartcard is one of the examples. Suppose there are some sensitive data stored in a smartcard, which has only very limited computation power. In order to send the sensitive data to a recipient in a secure and authenticated way, it should be encrypted using the recipient's public key and signed with the card owner's private key. To ensure timely and efficient delivery, it would be much better if part of the signcryption process could be done prior to know the data to be encrypted and the recipient's public key. Wireless sensor network (WSN) or mobile devices can be another example. Similar to smartcard, wireless sensors or mobile devices such as PDA or smart phone have only limited resources. It may take very long time, or even impossible to execute heavy computations on those tiny devices. Yet the data they process may be sensitive which is necessary to be encrypted and authenticated before sending off to the terminal stations. By using online/offline signcryption, the offline part (containing all heavy computation) can be done by a third party at the setup or manufacturing stage or when external power is connected. However, it is obvious that the data to be processed and the receiver's information is unknown at this stage.

In the above examples, the previous online/offline signcryption schemes (such as $[1,22]$ ) cannot be used, since they require the receiver's public key in the offline

stage. This maybe one of the very important reasons that previous online/offline signcryption schemes are not practical to be used in daily life applications. 


\subsection{Our Contributions}

In this paper, we make the following contributions.

1. We reformulate the notion of online/offline signcryption in the ID-based setting. We argue that it would be the best solution to provide authentication and confidentiality to smart cards or mobile devices for the following reasons. First, it combines the separate process of sign and encrypt into one "signcryption". Second, it even splits the signcryption process into online and offline stages, so that all the heavy computations can be performed in the offline stage, leaving only light operations such as hashing or integer multiplication to be done on tiny devices when the signcrypted message is known. Third, it is in the identity-based setting which gets rid of the costly process of certificate verification.

2. We present a concrete online/offline ID-based signcryption scheme, which does not require any heavy computation (such as pairing or elliptic curve multiplication) in the online stage. The security is proven using two assumptions, namely the Strong Diffie-Hellman (SDH) and Bilinear Diffie-Hellman Inversion (BDHI) assumptions in the random oracle model.

3. More importantly, unlike all other previous schemes ${ }^{1}$, our proposed scheme does not require the receiver's information (in our case, the identity) in the offline stage. The receiver's identity, together with the message to be signcrypted, are needed only in the online stage. This feature greatly increases the practicality of online/offline signcryption scheme. Our scheme is the first in the literature to allow this kind of flexibility.

4. When compared to the combination of online/offline ID-based encryption and online/offline ID-based signature, although the combination may achieve the same features as our scheme, efficiency is far more behind. Our scheme is about $30 \%-50 \%$ more efficient than any combination of the state of the art online/offline ID-based encryption and signature schemes.

\subsection{Organization}

The rest of the paper are organized as follows. We review some definitions in Section 2. It is followed by our proposed scheme in Section 3. We analyze the performance of our scheme in Section 4. Our paper is concluded in Section 5.

\footnotetext{
${ }^{1}$ Very recently, another identity-based online/offline signcryption scheme was proposed [17] in eprint. They have some comments to the preliminary version of our scheme [14] in eprint. However, the comments are not true in the current version. We also note that although the authors in [17] claimed that they are the first to propose such an ID-based online/offline signcryption with similar features as our scheme, it is not accurate. Our preliminary version [14] appeared before their scheme. It is, at most to say, two schemes with the same features were proposed almost at the same time. In addition, they do not contain any proof in the eprint version. We do not include their scheme for comparison.
} 


\section{Definitions}

\subsection{Pairings}

We briefly review the bilinear pairing. Let $\mathbb{G}$ and $\mathbb{G}_{T}$ be two multiplicative cyclic groups of prime order $q$. Let $g$ be a generator of $\mathbb{G}$, and $e$ be a bilinear map such that $e: \mathbb{G} \times \mathbb{G} \rightarrow \mathbb{G}_{T}$ with the following properties:

1. Bilinearity: For all $u, v \in \mathbb{G}$, and $a, b \in \mathbb{Z}, e\left(u^{a}, v^{b}\right)=e(u, v)^{a b}$.

2. Non-degeneracy: $e(g, g) \neq 1$.

3. Computability: It is efficient to compute $e(u, v)$ for all $u, v \in \mathbb{G}$.

\subsection{Intractability Assumption}

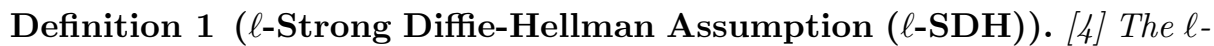
Strong Diffie-Hellman ( $\ell-S D H)$ problem in $\mathbb{G}$ is defined as follow: On input a $(\ell+1)$-tuple $\left(g, g^{\alpha}, g^{\alpha^{2}}, \cdots, g^{\alpha^{\ell}}\right) \in \mathbb{G}^{\ell+1}$, output a pair $\left(g^{\frac{1}{\alpha+c}}, c\right)$ where $c \in \mathbb{Z}_{q}^{*}$. We say that the $(t, \epsilon, \ell)-S D H$ assumption holds in $\mathbb{G}$ if no t-time algorithm has advantage at least $\epsilon$ in solving the $\ell-S D H$ problem in $\mathbb{G}$.

Definition 2 ( $\ell$-Bilinear Diffie-Hellman Inversion Assumption ( $\ell$-BDHI)). [3] The $\ell$-Diffie-Hellman ( $\ell-B D H I)$ problem in $\mathbb{G}$ is defined as follow: On input $a(\ell+1)$-tuple $\left(g, g^{\alpha}, g^{\alpha^{2}}, \cdots, g^{\alpha^{\ell}}\right) \in \mathbb{G}^{\ell+1}$, output $e(g, g)^{\frac{1}{\alpha}} \in \mathbb{G}_{T}$. We say that the $(t, \epsilon, \ell)-B D H I$ assumption holds in $\mathbb{G}$ if no $t$-time algorithm has advantage at least $\epsilon$ in solving the $\ell-B D H I$ problem in $\mathbb{G}$.

\subsection{Definition of Signcryption}

An ID-based online/offline signcryption scheme consists of the following six probabilistic polynomial time (PPT) algorithms:

- $($ param,$m s k) \leftarrow \operatorname{Setup}\left(1^{k}\right)$ takes a security parameter $k \in \mathbb{N}$ and generates param the global public parameters and $m s k$ the master secret key of the PKG.

- $D_{I D} \leftarrow$ Extract $\left(1^{k}\right.$, param, ms $\left.k, I D\right)$ takes a security parameter $k$, a global parameters param, a master secret key msk and an identity $I D$ to generate a secret key $D_{I D}$ corresponding to this identity.

$-\bar{\phi} \leftarrow$ Offline-Signcrypt $\left(1^{k}\right.$, param, $\left.D_{I D_{s}}\right)$ takes a security parameter $k$, a global parameters param, a secret key of the sender $D_{I D_{s}}$, to generate an offline ciphertext $\bar{\phi}$.

- $\phi \leftarrow$ Online-Signcrypt $\left(1^{k}\right.$, param, $\left.m, \bar{\phi}, I D_{r}\right)$ takes a security parameter $k$, a global parameters param, a message $m$, an identity of the receiver $I D_{r}$ where $I D_{s} \neq I D_{r}$, an offline ciphertext $\bar{\phi}$ to generate a ciphertext $\phi$.

- $(m, \sigma) / \perp \leftarrow \operatorname{De}$-Signcrypt $\left(1^{k}\right.$, param $\left., \phi, D_{I D_{r}}\right)$ takes a security parameter $k$, a global parameters param, a ciphertext $\phi$, a secret key of the receiver $D_{I D_{r}}$ to generate a message $m$ and a signature $\sigma$, or $\perp$ which indicates the failure of de-signcryption. 
- valid $/ \perp \leftarrow$ Verify $\left(1^{k}\right.$, param, $\left.m, \sigma, I D_{s}\right)$ takes a security parameter $k$, a global parameters param, a message $m$, a signature $\sigma$, an identity $I D_{s}$ to output valid of $\perp$ for an invalid signature.

For simplicity, we omit the notation of $1^{k}$ and param from the input arguments of the above algorithms in the rest of this paper. For correctness, if

$$
\begin{aligned}
\bar{\phi} & \leftarrow \text { Offline-Signcrypt }\left(D_{I D_{s}}\right) \\
\phi & \leftarrow \text { Online-Signcrypt }\left(m, \bar{\phi}, I D_{r}\right) \\
(\tilde{m}, \tilde{\sigma}) & \leftarrow \text { De-Signcrypt }\left(\phi, D_{I D_{r}}\right)
\end{aligned}
$$

we require that

$$
\begin{aligned}
\tilde{m} & =m \\
\operatorname{valid} & \leftarrow \operatorname{Verify}\left(\tilde{m}, \tilde{\sigma}, I D_{s}\right)
\end{aligned}
$$

Note that our definition differs from the one in [19] in the way where the offline signcrypt stage does not require the receiver's identity as input. In our definition, the receiver's identity is only required in the online signcrypt stage.

\subsection{Security of Signcryption}

Definition 3 (Confidentiality). An ID-based online/offline signcryption scheme is semantically secure against chosen ciphertext insider attack (SC-IND-CCA) if no PPT adversary has a non-negligible advantage in the following game:

1. The challenger runs Setup and gives the resulting param to adversary $\mathcal{A}$. It keeps msk secret.

2. In the first stage, $\mathcal{A}$ makes a number of queries to the following oracles which are simulated by the challenger:

(a) Extraction oracle: $\mathcal{A}$ submits an identity ID to the extraction oracle for the result of Extract $(m s k, I D)$.

(b) Signcryption oracle: $\mathcal{A}$ submits a sender identity I $D_{s}$, a receiver identity $I D_{r}$ and a message $m$ to the signcryption oracle for the result of Online-Signcrypt $\left(m\right.$, Offline-Signcrypt $\left.\left(D_{I D_{s}}\right), I D_{r}\right)$.

(c) De-signcryption oracle: $\mathcal{A}$ submits a ciphertext $\phi$ and a receiver identity $I D_{r}$ to the oracle for the result of De-Signcrypt $\left(\phi, D_{I D_{r}}\right)$. The result is made of a message and a signature if the de-signcryption is successful and the signature is valid under the sender's identity. Otherwise, a symbol $\perp$ is returned for rejection.

These queries can be asked adaptively. That is, each query may depend on the answers of previous ones.

3. $\mathcal{A}$ produces two messages $m_{0}, m_{1}$, two identities $I D_{s}^{*}, I D_{r}^{*}$ and a valid secret key $D_{I D_{s}^{*}}$ corresponding to $I D_{s}^{*}$. The challenger chooses a random bit $b \in$ $\{0,1\}$ and computes a signcryption ciphertext $\phi^{*}=$ Online-Signcrypt $\left(m_{b}\right.$, Offline-Signcrypt $\left.\left(D_{I D_{s}^{*}}\right), I D_{r}^{*}\right) . \phi^{*}$ is sent to $\mathcal{A}$. 
4. $\mathcal{A}$ makes a number of new queries as in the first stage with the restriction that it cannot query the de-signcryption oracle with $\left(\phi^{*}, I D_{r}^{*}\right)$ and the extraction oracle with I $D_{r}^{*}$.

5. At the end of the game, $\mathcal{A}$ outputs a bit $b^{\prime}$ and wins if $b^{\prime}=b$.

$\mathcal{A}$ 's advantage is defined as $\boldsymbol{A d \boldsymbol { d }}{ }^{I N D-C C A}(\mathcal{A})=\left|\operatorname{Pr}\left[b^{\prime}=b\right]-\frac{1}{2}\right|$.

Definition 4 (Unforgeability). A signcryption scheme is existentially unforgeable against chosen-message insider attack (SC-EUF-CMA) if no PPT adversary has a non-negligible advantage in the following game:

1. The challenger runs Setup and gives the resulting param to adversary $\mathcal{A}$. It keeps msk secret.

2. $\mathcal{A}$ makes a number of queries to the following oracles which are simulated by the challenger:

(a) Extraction oracle: $\mathcal{A}$ submits an identity ID to the extraction oracle for the result of Extract $(m s k, I D)$.

(b) Signcryption oracle: $\mathcal{A}$ submits a sender identity I $D_{s}$, a receiver identity $I D_{r}$ and a message $m$ to the signcryption oracle for the result of Online-Signcrypt $\left(m\right.$, Offline-Signcrypt $\left.\left(D_{I D_{s}}\right), I D_{r}\right)$.

(c) De-signcryption oracle: $\mathcal{A}$ submits a ciphertext $\phi$ and a receiver identity $I D_{r}$ to the oracle for the result of De-Signcrypt $\left(\phi, D_{I D_{r}}\right)$. The result is made of a message and a signature if the de-signcryption is successful and the signature is valid under the sender's identity. Otherwise, a symbol $\perp$ is returned for rejection.

These queries can be asked adaptively. That is, each query may depend on the answers of previous ones.

3. $\mathcal{A}$ produces a signcryption ciphertext $\phi^{*}$ and two identity $I D_{r}^{*}, I D_{s}^{*}$. $\mathcal{A}$ wins if

(a) De-Signcrypt $\left(\phi^{*}, D_{I D_{r}^{*}}\right)$ returns a tuple $\left(m^{*}, \sigma^{*}\right)$ such that valid $\leftarrow \operatorname{Verify}\left(m^{*}, \sigma^{*}, I D_{s}^{*}\right)$;

(b) No output of the signcryption oracle decrypts to $\left(m^{*}, \sigma^{*}\right)$ such that valid $\leftarrow$ $\operatorname{Verify}\left(m^{*}, \sigma^{*}, I D_{s}^{*}\right)$; and

(c) No extraction query was made on $I D_{s}^{*}$.

$\mathcal{A}$ 's advantage is defined as $\boldsymbol{A d \boldsymbol { d }}{ }^{E U F-C M A}(\mathcal{A})=\operatorname{Pr}[\mathcal{A}$ wins $]$

\section{The Proposed Online/Offline ID-Based Signcryption Scheme}

\subsection{Construction}

Let $\mathbb{G}$ and $\mathbb{G}_{T}$ be groups of prime-order $q$, and let $e: \mathbb{G} \times \mathbb{G} \rightarrow \mathbb{G}_{T}$ be the bilinear pairing. We use a multiplicative notation for the operation in $\mathbb{G}$ and $\mathbb{G}_{T}$.

Setup: The PKG selects a generator $g \in \mathbb{G}$ and randomly chooses $s \in_{R} \mathbb{Z}_{q}^{*}$. It $\overline{\text { sets } g_{1}}=g^{s}$ and $g_{2}=g_{1}^{s}$. Define $\mathcal{M}$ to be the message space. Let $n_{M}=|\mathcal{M}|$. Also 
let $n_{d}$ be the length of an identity, $H_{1}:\{0,1\}^{n_{d}} \rightarrow \mathbb{Z}_{q}^{*}, H_{2}:\{0,1\}^{*} \times \mathbb{G}_{T} \rightarrow \mathbb{Z}_{q}^{*}$ and $H_{3}:\{0,1\}^{*} \rightarrow\{0,1\}^{n_{M}}$ be some cryptographic hash functions. The public parameters param and master secret key msk are given by

$$
\text { param }=\left(\mathbb{G}, \mathbb{G}_{T}, q, g, g_{1}, g_{2}, \mathcal{M}, H_{1}, H_{2}, H_{3}\right) \quad m s k=s
$$

Extract: To generate a secret key for a user with identity $I D \in\{0,1\}^{n_{d}}$, the PKG computes:

$$
d_{I D} \leftarrow g^{\frac{1}{H_{1}(I D)+s}}
$$

The user also computes and stores $\overline{G_{I D}}=e\left(g^{H_{1}(I D)} g_{1}, g\right)$ for future use.

Offline-Signcrypt: The user with identity $I D_{s} \in\{0,1\}^{n_{d}}$, with secret key $d_{I D_{s}}$, at the offline stage first randomly generates $u, x, \alpha, \beta, \gamma, \delta \in_{R} \mathbb{Z}_{q}^{*}$ and computes:

$$
\begin{aligned}
U & \leftarrow d_{I D_{s}} g^{-u} \quad R \leftarrow\left(\overline{G_{I D_{s}}}\right)^{x} \\
T_{0} & \leftarrow\left(g^{\alpha H_{1}\left(I D_{s}\right)} g_{1}^{H_{1}\left(I D_{s}\right)+\gamma} g_{2}\right)^{x} \\
T_{1} & \leftarrow g^{x \beta^{-1} H_{1}\left(I D_{s}\right)} \quad T_{2} \leftarrow g_{1}^{x \delta^{-1}}
\end{aligned}
$$

Outputs the offline ciphertext $\bar{\phi}=\left(U, R, x, u, T_{0}, T_{1}, T_{2}, \alpha, \beta, \gamma, \delta\right)$.

Online-Signcrypt: At the online stage, to encrypt a message $m \in \mathcal{M}$ to a user with identity $I D_{r} \in\{0,1\}^{n_{d}}$ computes:

$$
\begin{gathered}
t_{1}^{\prime} \leftarrow \beta\left(H_{1}\left(I D_{r}\right)-\alpha\right) \bmod q \quad t_{2}^{\prime} \leftarrow \delta\left(H_{1}\left(I D_{r}\right)-\gamma\right) \bmod q \\
t \leftarrow h_{2} x+u \bmod q \quad c \leftarrow h_{3} \oplus m
\end{gathered}
$$

where $h_{2}=H_{2}\left(m, I D_{s}, R, T_{0}, T_{1}, T_{2}, t_{1}^{\prime}, t_{2}^{\prime}, U\right)$ and $h_{3}=H_{3}\left(R, T_{1}, T_{2}, U\right)$. Outputs the ciphertext $\phi=\left(U, t, c, T_{0}, T_{1}, T_{2}, t_{1}^{\prime}, t_{2}^{\prime}\right)$.

De-Signcrypt: To de-signcrypt $\phi$ using secret key $D_{I D_{r}}$, computes

$$
R \leftarrow e\left(T_{0} T_{1}^{t_{1}^{\prime}} T_{2}^{t_{2}^{\prime}}, d_{I D_{r}}\right) \quad m \leftarrow c \oplus H_{3}\left(R, T_{1}, T_{2}, U\right)
$$

and outputs $\left(m, \sigma, I D_{s}\right)$ where $\sigma=\left\{R, t, U, T_{0}, T_{1}, T_{2}, t_{1}^{\prime}, t_{2}^{\prime}\right\}$.

Verify: Computes $h_{2}=H_{2}\left(m, I D_{s}, R, T_{0}, T_{1}, T_{2}, t_{1}^{\prime}, t_{2}^{\prime}, U\right)$ and checks whether

$$
R^{h_{2}} \stackrel{?}{=} e\left(g^{t} U, g^{H_{1}\left(I D_{s}\right)} g_{1}\right) \cdot e(g, g)^{-1}
$$

Outputs valid if it is equal. Otherwise outputs $\perp$.

We note that the term $e(g, g)^{-1}$ can be pre-computed or published as part of the public parameter by the PKG. Thus the number of pairing required in the whole de-signcryption process is just 2, while there is no pairing required in either offline signcrypt or online signcrypt stage. 


\subsection{Security Analysis}

Theorem 1 (Confidentiality). If there is a SC-IND-CCA adversary $\mathcal{A}$ of the proposed scheme in Section 3 that succeeds with probability $\epsilon$, then there is a simulator $\mathcal{B}$ running in polynomial time that solves the $(\ell+1)-B D H I$ problem with probability at least

$$
\epsilon \cdot \frac{1}{q_{1}}\left(1-q_{s} \frac{q_{s}+q_{2}}{q}\right)\left(1-\frac{q_{d}}{q}\right)
$$

where $q_{1}, q_{2}, q_{3}, q_{s}, q_{d}$ are the number of queries allowed to the random oracle $H_{1}, H_{2}, H_{3}$, signcryption oracle and de-signcryption oracle respectively and we assume $q_{1}=\ell$.

Proof. Setup: Suppose $\mathcal{B}$ is given a random instance of the $(\ell+1)$-BDHI problem $\left(g, g^{\alpha}, g^{\alpha^{2}}, \ldots, g^{\alpha^{\ell}}, g^{\alpha^{\ell+1}}\right), \mathcal{B}$ runs $\mathcal{A}$ as a subroutine to output $e(g, g)^{\frac{1}{\alpha}} . \mathcal{B}$ sets up a simulated environment for $\mathcal{A}$ as follow.

$\mathcal{B}$ first randomly selects $\pi \in_{R}\left\{1, \ldots, q_{1}\right\}, I_{\pi} \in_{R} \mathbb{Z}_{q}^{*}$ and $w_{1}, \ldots, w_{\pi-1}$, $w_{\pi+1}, \ldots, w_{\ell} \in_{R} \mathbb{Z}_{q}^{*}$. For $i \in\{1, \ldots, \ell\} \backslash\{\pi\}$, it computes $I_{i}=I_{\pi}-w_{i}$. Construct a polynomial with degree $\ell-1$ as

$$
f(z)=\prod_{i=1, i \neq \pi}^{\ell}\left(z+w_{i}\right)
$$

to obtain $c_{0}, \ldots, c_{\ell-1} \in \mathbb{Z}_{q}^{*}$ such that $f(z)=\sum_{i=0}^{\ell-1} c_{i} z^{i}$. Then it sets generator $\hat{g}=g^{\sum_{i=0}^{\ell-1} c_{i} \alpha^{i}}=g^{f(\alpha)}$.

For $i \in\{1, \ldots, \ell\} \backslash\{\pi\}, \mathcal{B}$ expands $f_{i}(z)=\frac{f(z)}{\left(z+w_{i}\right)}=\sum_{j=0}^{\ell-2} d_{i, j} z^{j}$ to obtain $d_{i, 1}, \ldots, d_{i, \ell-2} \in \mathbb{Z}_{q}^{*}$ and sets

$$
\tilde{H}_{i}=g^{\sum_{j=0}^{\ell-2} d_{i, j} \alpha^{j}}=g^{f_{i}(\alpha)}=g^{\frac{f(\alpha)}{\alpha+w_{i}}}=\hat{g}^{\frac{1}{\alpha+w_{i}}}
$$

It computes the public key $g_{1}$ and $g_{2}$ as

$$
g_{1}=\hat{g}^{-\alpha} \hat{g}^{-I_{\pi}}=\hat{g}^{-\alpha-I_{\pi}} \quad g_{2}=\hat{g}^{\alpha^{2}} \hat{g}^{2 I_{\pi} \alpha} \hat{g}^{I_{\pi}^{2}}=\hat{g}^{\left(\alpha+I_{\pi}\right)^{2}}
$$

where $\hat{g}^{\alpha}=g^{\sum_{i=0}^{\ell-1} c_{i} \alpha^{i+1}}$ and $\hat{g}^{\alpha^{2}}=g^{\sum_{i=0}^{\ell-1} c_{i} \alpha^{i+2}}$ so that its unknown private key is implicitly set to $x=-\alpha-I_{\pi} \in \mathbb{Z}_{q}^{*}$. For all $i \in\{1, \ldots, \ell\} \backslash\{\pi\}$, we have $\left(I_{i},-\tilde{H}_{i}\right)=\left(I_{i}, \hat{g}^{\frac{1}{I_{i}+x}}\right)$.

Oracle Simulation: $\mathcal{B}$ first initializes a counter $\nu$ to 1 and starts $\mathcal{A}$. Throughout the game, we assume that $H_{1}$-queries are distinct, that the target identity $I D_{r}^{*}$ is submitted to $H_{1}$ at some point.

1. Random Oracle: For $H_{1}$-queries (we denote $I D_{\nu}$ the input of the $\nu^{t h}$ one of such queries), $\mathcal{B}$ answers $I_{\nu}$ and increments $\nu$. 
For $H_{2}$-queries on input $\left(m, I D_{s}, R, T_{0}, T_{1}, T_{2}, t_{1}^{\prime}, t_{2}^{\prime}, U\right)$ and $H_{3}$-queries on input $\left(R, T_{1}, T_{2}, U\right), \mathcal{B}$ returns the defined value if it exists and a randomly chosen $h_{2} \in_{R} \mathbb{Z}_{q}^{*}$ for $H_{2}$ and $h_{3} \in_{R}\{0,1\}^{n_{M}}$ for $H_{3}$ respectively, otherwise. $\mathcal{B}$ stores the information $\left\{h_{2},\left(m, I D_{s}, R, T_{0}, T_{1}, T_{2}, t_{1}^{\prime}, t_{2}^{\prime}, U, \gamma\right)\right\}$ in $L_{2}$, where $\gamma=R^{h_{2}} \cdot e(\hat{g}, \hat{g})$, and $\left\{h_{3},\left(R, T_{1}, T_{2}, U\right)\right\}$ in $L_{3}$.

2. Extraction Oracle: On input $I D_{\nu}$, if $\nu=\pi, \mathcal{B}$ aborts. Otherwise, it knows that $H_{1}\left(I D_{\nu}\right)=I_{\nu}$ and returns $-\tilde{H}_{\nu}=\hat{g}^{1 /\left(I_{\nu}+x\right)}$.

3. Signcryption Oracle: On input a plaintext $m$ and identities $\left(I D_{s}, I D_{r}\right)=$ $\left(I D_{\mu}, I D_{\nu}\right)$ for $\mu, \nu \in\left\{1, \ldots, q_{1}\right\}$, we observe that if $\mu \neq \pi, \mathcal{B}$ knows the sender's private key $d_{I D_{\mu}}=-\tilde{H}_{\mu}$ and can answer the query according to the specification of the algorithm. We thus assume $\mu=\pi$ and hence $\nu \neq \pi$. Also observe that $\mathcal{B}$ knows the receiver's private key $d_{I D_{\nu}}=-\tilde{H}_{\nu}$. The remaining task is to find a triple $\left(U, t, T_{0}, T_{1}, T_{2}, t_{1}^{\prime}, t_{2}^{\prime}, h\right)$ such that

$$
e\left(T, d_{I D_{\nu}}\right)^{h}=e\left(\hat{g}^{t} U, g_{I D_{\pi}}\right) \cdot e(\hat{g}, \hat{g})^{-1}
$$

where $T=T_{0} T_{1}^{t_{1}^{\prime}} T_{2}^{t_{2}^{\prime}}, g_{I D_{\pi}}=\hat{g}^{I_{\pi}} g_{1}$. To do so, $\mathcal{B}$ randomly generates $t, t^{\prime}, h, t_{1}^{\prime}, t_{2}^{\prime}, \tilde{t}_{1}, \tilde{t}_{2} \in_{R}$ $\mathbb{Z}_{q}^{*}$, computes

$$
\begin{gathered}
U=d_{I D_{\nu}}{ }^{t^{\prime}+t_{1}^{\prime} \tilde{t}_{1}+t_{2}^{\prime} \tilde{t}_{2}} \hat{g}^{-t} \quad R=e\left(T, d_{I D_{\nu}}\right) \\
T_{0}=g_{I D_{\pi}}{ }^{\frac{t^{\prime}}{h}} g_{I D_{\nu}}{ }^{-\frac{1}{h}} \quad T_{1}=g_{I D_{\pi}} \frac{\tilde{t}_{1}}{h} \quad T_{2}=g_{I D_{\pi}} \frac{\tilde{t}_{2}}{h}
\end{gathered}
$$

where $g_{I D_{\nu}}=\hat{g}^{I_{\nu}} g_{1}$, and back patching the hash value $H_{2}\left(m, I D_{\pi}, R, T_{0}, T_{1}, T_{2}, t_{1}^{\prime}, t_{2}^{\prime}, U\right)$ to $h$. These values satisfy equation (1) as

$$
\begin{aligned}
R^{h} & =e\left(T_{0} T_{1}^{t_{1}^{\prime}} T_{2}^{t_{2}^{\prime}}, d_{I D_{\nu}}\right)^{h} \\
& =e\left(g_{I D_{\pi}} t^{\prime}+t_{1}^{\prime} \tilde{t}_{1}+t_{2}^{\prime} \tilde{t}_{2} g_{I D_{\nu}}{ }^{-1}, d_{I D_{\nu}}\right) \\
& =e\left(g_{I D_{\pi}}, d_{I D_{\nu}}\right)^{t^{\prime}+t_{1}^{\prime} \tilde{t}_{1}+t_{2}^{\prime} \tilde{t}_{2}} \cdot e(\hat{g}, \hat{g})^{-1} \\
& =e\left(U \hat{g}^{t}, g_{I D_{\pi}}\right) \cdot e(\hat{g}, \hat{g})^{-1}
\end{aligned}
$$

and they are valid ciphertext tuples as the distribution of the simulated ciphertexts is the same as the one in the real protocol. The ciphertext $\phi=$ $\left(U, T_{0}, T_{1}, T_{2}, t_{1}^{\prime}, t_{2}^{\prime}, t, m \oplus H_{3}\left(R, T_{1}, T_{2}, U\right)\right)$ is returned.

4. De-signcryption Oracle: On input a ciphertext $\phi=\left(U, T_{0}, T_{1}, T_{2}, t_{1}^{\prime}, t_{2}^{\prime}, t, c\right)$ for identity (receiver) $I D_{r}=I D_{\nu}$, we assume that $\nu=\pi$ because otherwise $\mathcal{B}$ knows the receiver's private key $d_{I D_{\nu}}=-\tilde{H}_{\nu}$ and can normally run the decryption algorithm.

First, we note the following fact. Let $\tilde{x} \in \mathbb{Z}_{q}$ such that

$$
\begin{aligned}
d_{I D_{\mu}}{ }^{\tilde{x}} & =\hat{g}^{t} U d_{I D_{\mu}}{ }^{-1} \\
\left(\hat{g}^{\frac{1}{I_{\mu}+x}}\right)^{\tilde{x}} & =\hat{g}^{t} U \hat{g}^{\frac{-1}{I_{\mu}+x}} \\
\hat{g}^{\tilde{x}} & =\left(\hat{g}^{t} U\right)^{I_{\mu}+x} \hat{g}^{-1}
\end{aligned}
$$


Also let $T=T_{0} T_{1}^{t_{1}^{\prime}} T_{2}{ }^{t_{2}^{\prime}}, g_{I D_{\nu}}=\hat{g}^{I_{\nu}} g_{1}$, and $h=H_{2}\left(m, I D_{\mu}, R, \cdots\right)$ (which is yet unknown to $\mathcal{B}$ at this moment). As all valid ciphertext satisfies

$$
\begin{aligned}
R^{h} & =e\left(\hat{g}^{t} U, \hat{g}^{I_{\mu}+x}\right) \cdot e(\hat{g}, \hat{g})^{-1} \\
e\left(T^{h}, \hat{g}^{\frac{1}{I_{\nu}+x}}\right) & =e\left(\left(\hat{g}^{t} U\right)^{I_{\mu}+x}, \hat{g}\right) \cdot e\left(\hat{g}^{-1}, \hat{g}\right) \\
e\left(T^{\frac{h}{I_{\nu}+x}}, \hat{g}\right) & =e\left(\left(\hat{g}^{t} U\right)^{I_{\mu}+x} \hat{g}^{-1}, \hat{g}\right) \\
\Rightarrow T^{\frac{h}{I_{\nu}+x}} & =\left(\hat{g}^{t} U\right)^{I_{\mu}+x} \hat{g}^{-1}
\end{aligned}
$$

Let $\tilde{x}^{\prime} \in \mathbb{Z}_{q}$ such that

$$
\begin{aligned}
g_{I D_{\nu}} \tilde{x}^{\prime} & =T^{h} \\
\hat{g}^{\prime}\left(I_{\nu}+x\right) & =T^{h} \\
\hat{g}^{\prime} & =T^{\frac{h}{\nu_{\nu}+x}} \\
& =\left(\hat{g}^{t} U\right)^{I_{\mu}+x} \hat{g}^{-1}(\text { from equation(3)) } \\
& =\hat{g}^{\tilde{x}}(\text { from equation }(2)) \\
\Rightarrow \tilde{x}^{\prime} & =\tilde{x} \\
\Rightarrow \log _{d_{I D \mu}}\left(\hat{g}^{t} U{d_{I D_{\mu}}}^{-1}\right) & =\log _{g_{I D_{\nu}}}\left(T^{h}\right)
\end{aligned}
$$

From equation (4), we have

$$
e\left(T^{h}, d_{I D_{\mu}}\right)=e\left(g_{I D_{\nu}}, S \cdot d_{I D_{\mu}}{ }^{-1}\right)
$$

where $S=\hat{g}^{t} U$, which yields $e\left(T^{h}, d_{I D_{\mu}}\right)=e\left(g_{I D_{\nu}}, S\right) \cdot e\left(g_{I D_{\nu}}, d_{I D_{\mu}}\right)^{-1}$.

The query is handled by computing $\gamma=e\left(S, \hat{g}^{I_{\mu}} g_{1}\right)$, and search through the list $L_{2}$ for entries of the form $\left(m_{i}, R_{i}, h_{2, i}, \cdots, \gamma\right)$ indexed by $i \in\left\{1, \ldots, q_{2}\right\}$. If none is found, $\phi$ is rejected. Otherwise, each one of them is further examined: for the corresponding indexes, $\mathcal{B}$ checks if

$$
\frac{e\left(T, d_{I D_{\mu}}\right)^{h_{2, i}}}{e\left(S, g_{I D_{\nu}}\right)}=e\left(g_{I D_{\nu}}, d_{I D_{\mu}}\right)^{-1}
$$

meaning that equation (5) is satisfied. If the unique $i \in\left\{1, \ldots, q_{2}\right\}$ satisfying equation (6) is detected, the matching pair $\left(m_{i}, h_{2, i}, S\right)$ is returned. Otherwise $\phi$ is rejected.

Challenge: $\mathcal{A}$ outputs messages $\left(m_{0}, m_{1}\right)$ and identities $I D^{*}$ for which it never obtained $I D^{*}$ s private key. If $I D^{*} \neq I D_{\pi}, \mathcal{B}$ aborts. Otherwise it randomly selects $t, t^{\prime}{ }_{1}, t^{\prime}{ }_{2}, \tilde{t}_{0}, \tilde{t}_{1}, \tilde{t}_{2} \in_{R} \mathbb{Z}_{q}^{*}, c \in \in_{R}\{0,1\}^{n_{m}}$ and $U \in_{R} \mathbb{G}$. Computes $T_{0}=$ $\hat{g}^{\tilde{t}_{0}}, T_{1}=\hat{g}^{\tilde{t}_{1}}, T_{2}=\hat{g}^{\tilde{t}_{2}}$ to return the challenge ciphertext $\phi^{*}=\left(U, t, T_{0}, T_{1}\right.$, 
$\left.T_{2}, t_{1}^{\prime}, t_{2}^{\prime}, c\right)$. Let $\xi=\tilde{t}_{0}+t_{1}^{\prime} \tilde{t}_{1}+t_{2}^{\prime} \tilde{t}_{2}$ and $T=\hat{g}^{-\xi}$. Since $x=-\alpha-I_{\pi}$, we let $\rho=\frac{\xi}{\alpha\left(I_{\mu}-\alpha-I_{\pi}\right)}=-\frac{\xi}{\left(I_{\pi}+x\right)\left(I_{\mu}+x\right)}$, we can check that

$$
\begin{aligned}
T & =\hat{g}^{-\xi}=\hat{g}^{-\alpha\left(I_{\mu}-\alpha-I_{\pi}\right) \rho} \\
& =\hat{g}^{\left(I_{\pi}+x\right)\left(I_{\mu}+x\right) \rho} \\
& =\hat{g}^{\left(I_{\pi} I_{\mu}+\left(I_{\mu}+I_{\pi}\right) x+x^{2}\right) \rho}
\end{aligned}
$$

$\mathcal{A}$ cannot recognize that $\phi^{*}$ is not a proper ciphertext unless it queries $H_{2}$ or $H_{3}$ on $e\left(\hat{g}^{I_{\mu}} g_{1}, \hat{g}\right)^{\rho}$. Along the guess stage, its view is simulated as before and its output is ignored. Standard arguments can show that a successful $\mathcal{A}$ is very likely to query $H_{2}$ or $H_{3}$ on the input $e\left(g_{I D_{\mu}}, \hat{g}\right)^{\rho}$ if the simulation is indistinguishable from a real attack environment.

Output Calculation: $\mathcal{B}$ fetches a random entry $\left(m, R, T_{0}, T_{1}, T_{2}, t_{1}^{\prime}, t_{2}^{\prime}, U, h_{2}\right)$ or $\left(R, T_{1}, T_{2}, U, \cdot\right)$ from the lists $L_{2}$ or $L_{3}$. With probability $1 /\left(2 q_{2}+q_{3}\right)$, the chosen entry will contain the right element

$$
R=e\left(g_{I D_{\mu}}, \hat{g}\right)^{\rho}=e(\hat{g}, \hat{g})^{-\xi /\left(I_{\pi}+x\right)}=e(g, g)^{f(\alpha)^{2} \xi / \alpha}
$$

where $f(z)=\sum_{i=0}^{\ell-1} c_{i} z^{i}$ is the polynomial for which $\hat{g}=g^{f(\alpha)}$. The $(\ell+1)$-BDHI solution can be extracted by computing

$$
\begin{aligned}
& \left(\frac{R^{1 / \xi}}{e\left(g^{\sum_{i=0}^{\ell-2} c_{i+1} \alpha^{i}}, g^{c_{0}}\right) e\left(g^{\sum_{j=0}^{\ell-2} c_{j+1} \alpha^{j}}, \hat{g}\right)}\right)^{1 / c_{0}^{2}} \\
= & \left(\frac{e(g, g)^{f(\alpha)^{2} / \alpha}}{e(g, g)^{c_{0}\left(c_{1}+c_{2} \alpha+c_{3} \alpha^{2}+\ldots c_{\ell-1} \alpha^{\ell-2}\right)} e(g, g)^{f(\alpha)\left(c_{1}+c_{2} \alpha+c_{3} \alpha^{2}+\ldots c_{\ell-1} \alpha^{\ell-2}\right)}}\right)^{1 / c_{0}^{2}} \\
= & \left(\frac{e(g, g)^{f(\alpha)^{2} / \alpha}}{e(g, g)^{\frac{c_{0}\left(c_{1} \alpha+c_{2} \alpha^{2}+\ldots c_{\ell-1} \alpha^{\ell-1}\right)+f(\alpha)\left(c_{1} \alpha+c_{2} \alpha^{2}+\ldots c_{\ell-1} \alpha^{\ell-1}\right)}{\alpha}}}\right)^{1 / c_{0}^{2}} \\
= & e(g, g)^{\frac{f(\alpha)^{2}-\left(c_{1} \alpha+c_{2} \alpha^{2}+\ldots c_{\ell-1} \alpha^{\ell-1}\right)\left(c_{0}+f(\alpha)\right)}{c_{0}{ }^{2} \alpha}} \\
= & e(g, g)^{\frac{c_{0}^{2}}{c_{0}^{2} \alpha}} \\
= & e(g, g)^{1 / \alpha}
\end{aligned}
$$

Probability Analysis: $\mathcal{B}$ only fails in providing a consistent simulation because one of the following independent events happen:

- $E_{1}: \mathcal{A}$ does not choose to be challenged on $I D_{\pi}$.

- $E_{2}:$ A key extraction query is made on $I D_{\pi}$.

- $E_{3}: \mathcal{B}$ aborts in a Signcryption query because of a collision on $H_{2}$.

- $E_{4}: \mathcal{B}$ rejects a valid ciphertext at some point of the game. 
We have $\operatorname{Pr}\left[\neg E_{1}\right]=1 / q_{1}$ and $\neg E_{1}$ implies $\neg E_{2}$. Also observe that $\operatorname{Pr}\left[E_{3}\right] \leq$ $q_{s}\left(q_{s}+q_{2}\right) / q$ and $\operatorname{Pr}\left[E_{4}\right] \leq q_{d} / q$. Combining together, the overall successful probability $\operatorname{Pr}\left[\neg E_{1} \wedge \neg E_{3} \wedge \neg E_{4}\right]$ is at least

$$
\frac{1}{q_{1}}\left(1-q_{s} \frac{q_{s}+q_{2}}{q}\right)\left(1-\frac{q_{d}}{q}\right)
$$

Theorem 2 (Unforgeability). If there is an SC-UEF-CMA adversary $\mathcal{A}$ of the proposed scheme in Section 3 that succeeds with probability $\epsilon$, then there is a simulator $\mathcal{B}$ running in polynomial time that solves the $\ell+1-S D H$ problem with probability at least

$$
\epsilon^{2} \cdot \frac{1}{q_{1} q_{2}}\left(1-q_{s} \frac{q_{s}+q_{2}}{q}\right)\left(1-\frac{q_{d}}{q}\right)
$$

where $q_{1}, q_{2}, q_{3}, q_{s}, q_{d}$ are the number of queries allowed to the random oracle $H_{1}, H_{2}, H_{3}$, signcryption oracle and de-signcryption oracle respectively and we assume $q_{1}=\ell$.

Proof. Setup: Suppose $\mathcal{B}$ is given a random instance of the $(\ell+1)-\mathrm{SDH}$ problem $\left(g, g^{\alpha}, \overline{g^{\alpha^{2}}}, \ldots, g^{\alpha^{\ell}}, g^{\alpha^{\ell+1}}\right), \mathcal{B}$ runs $\mathcal{A}$ as a subroutine to output $\left(c, g^{\frac{1}{c+\alpha}}\right) . \mathcal{B}$ sets up a simulated environment for $\mathcal{A}$ as follow.

$\mathcal{B}$ first randomly selects $\pi \in_{R}\left\{1, \ldots, q_{1}\right\}, I_{\pi} \in \in_{R} \mathbb{Z}_{q}^{*}$ and $w_{1}, \ldots, w_{\pi-1}$, $w_{\pi+1}, \ldots, w_{\ell} \in_{R} \mathbb{Z}_{q}^{*}$. Construct a polynomial with degree $\ell-1$ as

$$
f(z)=\prod_{i=1, i \neq \pi}^{\ell}\left(z+w_{i}\right)
$$

to obtain $c_{0}, \ldots, c_{\ell-1} \in \mathbb{Z}_{q}^{*}$ such that $f(z)=\sum_{i=0}^{\ell-1} c_{i} z^{i}$. Then it sets generator $\hat{g}=g^{\sum_{i=0}^{\ell-1} c_{i} \alpha^{i}}=g^{f(\alpha)}$.

For $i \in\{1, \ldots, \ell\} \backslash\{\pi\}, \mathcal{B}$ expands $f_{i}(z)=f(z) /\left(z+w_{i}\right)=\sum_{j=0}^{\ell-2} d_{i, j} z^{j}$ to obtain $d_{i, 1}, \ldots, d_{i, \ell-2} \in \mathbb{Z}_{q}^{*}$ and sets

$$
\tilde{H}_{i}=g^{\sum_{j=0}^{\ell-2} d_{i, j} \alpha^{j}}=g^{f_{i}(\alpha)}=g^{\frac{f(\alpha)}{\alpha+w_{i}}}=\hat{g}^{\frac{1}{\alpha+w_{i}}}
$$

It computes the public key $g_{1}$ and $g_{2}$ as

$$
g_{1}=\hat{g}^{\alpha} \quad g_{2}=\hat{g}^{\alpha^{2}}
$$

where $\hat{g}^{\alpha}=g^{\sum_{i=0}^{\ell-1} c_{i} \alpha^{i+1}}$ and $\hat{g}^{\alpha^{2}}=g^{\sum_{i=0}^{\ell-1} c_{i} \alpha^{i+2}}$ so that its unknown private key is implicitly set to $x=\alpha$. For all $i \in\{1, \ldots, \ell\} \backslash\{\pi\}$, we have $\left(I_{i}, \tilde{H}_{i}\right)=$ $\left(w_{i}, \hat{g}^{\frac{1}{w_{i}+\alpha}}\right)$.

Oracle Queries are answered in the same way as in Theorem 1. 
Output Calculation: $\mathcal{A}$ has produced a forged ciphertext $\left(U, T_{0}, T_{1}, T_{2}, t_{1}^{\prime}, t_{2}^{\prime}, t_{1}, c\right)$, a sender identity $I D_{s}$ and a receiver identity $I D_{r}$. If $I D_{s} \neq I D_{\pi}, \mathcal{B}$ aborts. Otherwise $\mathcal{B}$ uses $D_{I D_{r}}$ to decrypt and gets $R$ and $m^{*}$.

We denote $h_{1}$ for the reply of the $H_{2}$ query on $\left(m^{*}, I D_{\pi}, R, T_{0}, T_{1}, T_{2}, t_{1}^{\prime}, t_{2}^{\prime}, U\right)$. $\mathcal{B}$ rewinded to the point just before making this particular query. This time $\mathcal{B}$ supplies to a different value $h_{2} \neq h_{1}$ to this query. $\mathcal{A}$ produced another forged ciphertext $\left(U, \hat{T}_{0}, T_{1}, T_{2}, \hat{t_{1}^{\prime}}, \hat{t_{2}^{\prime}}, \hat{t}, \hat{c}\right)$ based on $h_{2}$. Note that $\left(U, T_{1}, T_{2}\right)$ are the same in both ciphertext as they are inputs to the $H_{2}$ query. $R$ and $m^{*}$ are also the same, as they are also one of the inputs to the $H_{2}$ query. By rewinding to the point just before making this particular query does not change the input values, but only the output values. If both forgeries satisfy equation (1), we obtain the relations

$$
e\left(S_{1}, g_{I D_{\pi}}\right)^{\frac{1}{h_{1}}} e(\hat{g}, \hat{g})^{-\frac{1}{h_{1}}}=e\left(S_{2}, g_{I D_{\pi}}\right)^{\frac{1}{h_{2}}} e(\hat{g}, \hat{g})^{-\frac{1}{h_{2}}}
$$

where $S_{1}=\hat{g}^{t} U, S_{2}=\hat{g}^{\hat{t}} U$ and $g_{I D_{\pi}}=\hat{g}^{H_{1}\left(I D_{\pi}\right)} g_{1}=\hat{g}^{I_{\pi}+\alpha}$. Then, it comes that

$$
e\left(S_{1}^{\frac{h_{2}}{h_{2}-h_{1}}} S_{2}^{-\frac{h_{1}}{h_{2}-h_{1}}}, g_{I D_{\pi}}\right)=e(\hat{g}, \hat{g})
$$

Let $\tilde{T}=S_{1}^{\frac{h_{2}}{h_{2}-h_{1}}} S_{2}{ }^{-\frac{h_{1}}{h_{2}-h_{1}}}=\hat{g}^{\frac{1}{T_{\pi}+\alpha}}$. From $\tilde{T}, \mathcal{B}$ can proceed as in [4] to extract $\sigma^{*}=g^{\frac{1}{T_{\pi}+\alpha}}:$ it first obtains $\gamma_{-1}, \gamma_{0}, \ldots, \gamma_{q-2} \in \mathbb{Z}_{q}^{*}$ for which $f(z) /\left(z+I_{\pi}\right)=$ $\frac{\gamma-1}{z+I_{\pi}}+\sum_{i=1}^{\ell-2} \gamma_{i} z^{*}$ and computes

$$
\sigma^{*}=\left(\tilde{T} g^{-\sum_{i=0}^{\ell-2} \gamma_{i} \alpha^{i}}\right)^{\frac{1}{\gamma-1}}=g^{\frac{1}{1 \pi+\alpha}}
$$

and returns the pair $\left(I_{\pi}, \sigma^{*}\right)$ as a result.

Probability Analysis is similar to the one in Theorem 1. In addition, there is a rewind here, with successful probability $\epsilon / q_{2}$. Combine together, the overall successful probability is at least

$$
\epsilon^{2} \cdot \frac{1}{q_{1} q_{2}}\left(1-q_{s} \frac{q_{s}+q_{2}}{q}\right)\left(1-\frac{q_{d}}{q}\right)
$$

\section{Performance Analysis}

The performance of our scheme is comparable to previous non-identity based online/offline signcryption scheme, such as $[1,22]$. Yet they need to fix the receiver's public key in the offline stage but we allow it to be known only in the online stage.

In terms of functionality, our scheme can be replaced by an online/offline identity-based encryption (OOIBE) (such as $[11,15]$ ) plus an online/offline identitybased signature (OOIBS) (such as [10]) to obtain the same features. However, 
the efficiency of our scheme highly surpasses the combination of an OOIBE and OOIBS. The advantages are shown in the following table. In the comparison, we assume that $|\mathbb{G}|=160 \mathrm{bits},|q|=160 \mathrm{bits},\left|\mathbb{G}_{T}\right|=1024$ bits and $|\mathcal{M}|=|q|=160$ bits. We denote by $E$ the point multiplication in $\mathbb{G}$ or $\mathbb{G}_{T}, M E$ the multi-point multiplication in $\mathbb{G}$ or $\mathbb{G}_{T}$ (which costs about 1.3 times more than a single point multiplication), $M$ the point addition in $\mathbb{G}$ or $\mathbb{G}_{T}$ and $m_{c}$ the modular computation in $\mathbb{Z}_{q}$.

\begin{tabular}{|l||c|c|c|c|}
\hline & $\begin{array}{c}\text { GMC-1 } \\
+ \text { OOIBS }\end{array}$ & $\begin{array}{c}\text { GMC-2 } \\
+ \text { OOIBS }\end{array}$ & $\begin{array}{c}\text { LZ } \\
+ \text { OOIBS }\end{array}$ & Our scheme \\
\hline \hline Offline computation & $6 E+2 M E$ & $5 E+2 M E$ & $5 E+1 M E$ & $4 E+1 M E$ \\
\hline Online computation & $1 M+3 m_{c}$ & $1 M+3 m_{c}$ & $4 m_{c}$ & $3 m_{c}$ \\
\hline Offline storage (bits) & 2944 & 5376 & 2944 & 2624 \\
\hline Ciphertext length (bits) & 3104 & 7424 & 2080 & 1280 \\
\hline $\begin{array}{c}\text { Number of pairing for } \\
\text { decryption + verification }\end{array}$ & 9 & 4 & 5 & 2 \\
\hline Security model & selective ID & standard & random oracle & random oracle \\
\hline
\end{tabular}

Table 1. Comparison of computation cost and size

Currently there are just 3 OOIBE schemes that allow the intended receiver's identity to be unknown in the offline stage. We use GMC-1 and GMC-2 to denote the first two in [11] and LZ to denote the one in [15]. For OOIBS, there is only one concrete scheme by $\mathrm{Xu}$ et al. [20]. However it was proven insecure by $\mathrm{Li}$ et al. [12] later. We use the generic construction by Galindo et al. [10]. The generic construction requires one public key based signature scheme and one online/offline signature scheme. The underlying signature schemes we use are from [6] (random oracle) and [4] (without random oracle) and the underlying online/offline signature scheme we use is from [5]. All these signature schemes are the most efficient one in the state of the art within their respective security model.

From the above table, we can see that our scheme achieves the least computation and the smallest size in both offline and online stage, when compare to the combinations of OOIBE and OOIBS.

\section{Conclusion}

In this paper, we redefined the notion "online/offline ID-based signcryption" and provided a scheme that realizes it. Our construction is very efficient in a sense that it does not require any pairing operation in offline and online signcryption stages. Furthermore, we do not require the receiver's information (in our case, identity) in the offline signcryption stage. It is the first in the literature to remove such requirement. Without this restriction, our scheme is more flexible and practical. Our scheme is particularly suitable to provide authentication 
and confidentiality to power-constrained communication devices. We believe our proposed scheme may provide a practical solution in secure and authenticated transaction for smart cards or mobile devices such as smart phone.

\section{References}

1. J. H. An, Y. Dodis, and T. Rabin. On the Security of Joint Signature and Encryption. In Proc. EUROCRYPT 2002, volume 2332 of Lecture Notes in Computer Science, pages 83-107. Springer-Verlag, 2002.

2. P. Barreto, B. Libert, N. McCullagh, and J. Quisquater. Efficient and provableysecure identity-based signature and signcryption from bilinear maps. In AsiaCrypt 2005, volume 3788 of Lecture Notes in Computer Science, pages 515-532. SpringerVerlag, 2005.

3. D. Boneh and X. Boyen. Efficient Selective-ID Secure Identity-Based Encryption Without Random Oracles. In Proc. EUROCRYPT 2004, volume 3027 of Lecture Notes in Computer Science, pages 223-238. Springer-Verlag, 2004.

4. D. Boneh and X. Boyen. Short Signatures Without Random Oracles. In Proc. EUROCRYPT 2004, volume 3027 of Lecture Notes in Computer Science, pages 56-73. Springer-Verlag, 2004.

5. D. Boneh and X. Boyen. Short signatures without random oracles and the sdh assumption in bilinear groups. J. Cryptology, 21(2):149-177, 2008.

6. D. Boneh, B. Lynn, and H. Shacham. Short Signatures from the Weil Pairing. In Proc. ASIACRYPT 2001, volume 2248 of Lecture Notes in Computer Science, pages 514-532. Springer-Verlag, 2001.

7. X. Boyen. Multipurpose Identity-Based Signcryption (A Swiss Army Knife for Identity-Based Cryptography). In Proc. CRYPTO 2003, volume 2729 of Lecture Notes in Computer Science, pages 383-399. Springer-Verlag, 2003.

8. L. Chen and J. Malone-Lee. Improved Identity-Based Signcryption. In Proc. PKC 2005, volume 3386 of Lecture Notes in Computer Science, pages 362-379. SpringerVerlag, 2005.

9. S. Even, O. Goldreich, and S. Micali. On-line/offline digital signatures. In Proc. CRYPTO 89, volume 2442 of Lecture Notes in Computer Science, pages 263-277. Springer-Verlag, 1989.

10. D. Galindo, J. Herranz, and E. Kiltz. On the generic construction of identity-based signatures with additional properties. In ASIACRYPT, volume 4284 of Lecture Notes in Computer Science, pages 178-193. Springer, 2006.

11. F. Guo, Y. Mu, and Z. Chen. Identity-based online/offline encryption. In Financial Cryptography and Data Security '08, volume 5143 of Lecture Notes in Computer Science, pages 247-261. Springer-Verlag, 2008.

12. F. Li, M. Shirase, and T. Takagi. On the security of online/offline signatures and multisignatures from acisp'06. In CANS '08, volume 5339 of Lecture Notes in Computer Science, pages 108-119. Springer-Verlag, 2008.

13. B. Libert and J.-J. Quisquater. New Identity Based Signcryption Schemes from Pairings. IEEE Information Theory Workshop 2003, pages 155-158, 2003.

14. J. K. Liu, J. Baek, and J. Zhou. Online/offline identity-based signcryption revisited. Cryptology ePrint Archive, Report 2010/274, 2010. http://eprint.iacr.org/.

15. J. K. Liu and J. Zhou. An efficient identity-based online/offline encryption scheme. In $A C N S$, volume 5536 of Lecture Notes in Computer Science, pages 156-167. Springer, 2009. 
16. J. Malone-Lee. Identity-Based Signcryption. Cryptology ePrint Archive, Report 2002/098, 2002. http://eprint.iacr.org/.

17. S. S. D. Selvi, S. S. Vivek, and C. P. Rangan. Identity based online/offline signcryption scheme. Cryptology ePrint Archive, Report 2010/376, 2010. http://eprint.iacr.org/.

18. A. Shamir. Identity-Based Cryptosystems and Signature Schemes. In Proc. CRYPTO 84, volume 196 of Lecture Notes in Computer Science, pages 47-53. Springer-Verlag, 1984.

19. D. Sun, Y. Mu, and W. Susilo. A generic construction of identity-based online/offline signcryption. In ISPA, pages 707-712. IEEE, 2008.

20. S. Xu, Y. Mu, and W. Susilo. Online/offline signatures and multisignatures for AVOD and DSR routing security. In ACISP '06, volume 4058 of Lecture Notes in Computer Science, pages 99-110. Springer-Verlag, 2006.

21. Z. Xu, G. Dai, and D. Yang. An efficient online/offline signcryption scheme for MANET. In AINA Workshop '07, pages 171-176. IEEE Computer Society, 2007.

22. F. Zhang, Y. Mu, and W. Susilo. Reducing security overhead for mobile networks. In AINA Workshop '05, pages 398-403. IEEE Computer Society, 2005.

23. Y. Zheng. Digital Signcryption or How to Achieve Cost(Signature \& Encryption) $<<$ Cost(Signature) + Cost(Encryption). In Proc. CRYPTO 9\%, volume 1294 of Lecture Notes in Computer Science, pages 165-179. Springer-Verlag, 1997. 